\title{
Loss of the nucleoporin Aladin in central nervous system and fibroblasts of Allgrove
}

\section{Syndrome}

Giacomo Bitetto, ${ }^{1}$ Dario Ronchi, ${ }^{1}$ Sara Bonato, ${ }^{1}$ Alessandra Pittaro, ${ }^{2}$ Giacomo Monzio Compagnoni, ${ }^{1}$ Andreina Bordoni, ${ }^{1}$ Sabrina Salani, ${ }^{1}$ Emanuele Frattini, ${ }^{1}$ Gianluca Lopez, ${ }^{2}$ Fulvia Milena Cribiù, ${ }^{2}$ Stefania Corti, ${ }^{1}$ Giacomo P. Comi, ${ }^{1}$ Nereo Bresolin, ${ }^{1}$ Alessio Di Fonzo ${ }^{1 *}$

${ }^{1}$ IRCCS Foundation Ca' Granda Ospedale Maggiore Policlinico, Neurology Unit, Milan, Italy; Dino Ferrari Center, Neuroscience Section, Department of Pathophysiology and Transplantation, University of Milan, Milan, Italy

${ }^{2}$ IRCCS Foundation Ca’ Granda Ospedale Maggiore Policlinico, Division of Pathology, University of Milan, Milan, Italy

${ }^{*}$ Correspondence: Alessio Di Fonzo

Email:Alessio.difonzo@policlinico.mi.it

Number: +39 3311599251

FAX: 0255033800

Address: IRCCS Ca’ Granda Ospedale Maggiore Policlinico, Via Francesco Sforza 35, 20122, Milan, Italy

\begin{abstract}
Allgrove syndrome (AS) is a rare disease with broad neurological involvement. Neurodegeneration can affect spinal motor neurons, Purkinje cells, striatal neurons, and the autonomic system. The mechanisms that lead to neuronal loss are still unclear. Recessive mutations in the $A A A S$ gene affect
\end{abstract}


the encoded protein Aladin, which would normally localize to the cytoplasmic face of the nuclear membrane as part of the nuclear pore complex (NPC). While the NPC is known to be a key factor for nucleo-cytoplasmic transport, the precise role of Aladin has not been elucidated yet. Here, we explored the consequences of the homozygous $A A A S$ mutation c.464G>A (p.R155H) in central nervous system tissues and fibroblasts of a novel AS patient presenting motor neuron disease, cerebellar ataxia, and autonomic dysfunction. Neuropathological analyses showed severe loss of motor neurons and Purkinje cells, with significant reduction in the perinuclear expression of Aladin. A reduced amount of protein was detected in the nuclear membrane fraction of the patient's brain. RNA analysis revealed a significant reduction of the transcript AAAS-1, while the AAAS-2 transcript was upregulated in fibroblasts. To our knowledge, this is the first study to demonstrate the effects of $A A A S$ mutations in human central nervous system.

\section{Introduction}

Allgrove syndrome (AS) (MIM 231550) is a rare, autosomal recessive, systemic disease, caused by mutations in the AAAS gene (1). This gene encodes for the WD-repeat-containing protein Aladin, a highly conserved nucleoporin (Nup), whose complex function is still under debate (2). The disease was named after Dr. Jeremy Allgrove, who first described a pair of siblings with a multisystem syndrome characterized by addisonian glucocorticoid deficiency, achalasia of the cardia, and alacrimia (3). In addition to this triad, for which AS is also known as "triple A syndrome", other clinical features have been described as part of the clinical spectrum, including dysautonomia, peripheral neuropathy, pyramidal and cerebellar signs, mental disability, optic atrophy, muscle waste, and skeletal abnormalities (4). For this reason, broader nomenclatures have been proposed which would account for prominent neurologic involvement (e.g., "4A" and " $5 \mathrm{~A}$ " syndrome to include autonomic dysfunction and/or amyotrophy) (5). AS typically presents in the first decade of 
life (80\% of cases), with a median age at onset of 5 years, and subsequently progresses along heterogenous courses (6).

The AAAS gene is expressed as two differentially spliced isoforms, which differ in the inclusion of exon 6 (7). The encoded protein is one of the Nups forming the nuclear pore complex (NPC), the largest protein complex in eukaryotic cells (8). The NPC maintains proper communication between the nucleus and the cytoplasm, shuttling proteins and RNAs across the nuclear membrane (9). Aladin lies on the cytoplasmic face of the complex, however its precise role in transport is still unknown (10). Defects in nucleo-cytoplasmic transport (NCT) and Nups have been implicated in pathogenetic mechanisms of several neurodegenerative diseases. In particular, repeat expansion disorders (i.e., amyotrophic lateral sclerosis/frontotemporal dementia complex with C9orf72 hexanucleotide expansion, Huntington's disease, and spinal cerebellar ataxias) and other neurodegenerative diseases with aberrant protein accumulations (i.e., TDP43, FUS, and TAU in frontotemporal dementia and Alzheimer's disease) are associated with mislocalization of specific Nups and NPC dysfunction (11)(12)(13). Moreover, mutations in several Nups have been demonstrated to lead to neuronal loss in various conditions (14), suggesting that NCT is of particular relevance for neuronal function and survival (15)(16).

Interestingly, mutations in the Nup Aladin are also associated with neuronal loss, which manifests in the core clinical presentation with signs of motor neuron, cerebellar, and striatal impairment. However, exactly how Aladin dysfunction triggers neurodegeneration and the specific consequences of $A A A S$ mutations at the cellular level remain obscure.

In this study, we explored the effects of an AAAS gene mutation in the brain and spinal cord, as well as in fibroblasts of a patient with late-onset AS displaying motor neuron disease, cerebellar ataxia, bulbar signs, and peripheral neuropathy. 


\section{Results}

\section{$\underline{\text { Clinical report }}$}

The patient was a 75-year-old male known to our institution for achalasia, with onset at 47 years of age, and who presented to our attention for progressive gait impairment since the age of 59. The neurological examination of cranial nerves showed pupillary miosis and bilateral absence of pupillary reflex, hypophonic and nasal speech, and slight tongue atrophy. Oropharyngeal motility and other cranial functions were unharmed. Muscle tone was mildly increased in the upper limbs, with generalized distal hypotrophy. Deep tendon reflexes were symmetrical and brisk, Hoffman sign was positive bilaterally. Lower limbs were hypotrophic, with diffuse and symmetrical hyperreflexia, except for Achilles reflexes, which were absent; plantar reflex was abnormal bilaterally, and both feet presented characteristic arching (pes cavus). No sensory or strength impairment was detected in the limbs. The gait appeared ataxic, with a tendency to retropulsion, and was possible only with double support.

Upon collection of medical history, the patient recalled past episodes of loss of consciousness while transferring from lying to standing, suggesting orthostatic hypotension, which was confirmed by the clinical evaluation. The patient had also presented episodes of symptomatic hypoglycemia, which were ascribed by endocrinological workup to adrenal insufficiency requiring substitutive steroidal therapy. He died at 78 years due to aspiration pneumonia.

Brain MRI exhibited atrophy of the cerebellum, frontal cortex and basal ganglia.

EMG showed chronic and active neurogenic damage suggestive of motor neuron involvement. ENG displayed features of axonal sensitive and motor neuropathy.

The biopsy of the gastrocnemius muscle showed tissue degeneration, with substitution of muscle fibers with fibrous-adipose material. The biopsy of sural and ilioinguinal nerves showed a significant reduction of myelinic fibers associated with connective-endoneurial substitution.

Schirmer's test revealed hypolacrimation. (Figure 1) 


\section{$\underline{\text { Molecular studies }}$}

Sanger sequencing revealed the homozygous c.464G>A (p.R155H) substitution. (Figure 1)

The variant was absent in more than 1000 Italian exomes and in the $1000 \mathrm{G}$ and Exac databases, and was predicted to be pathogenic by Mutation Taster (http://www.mutationtaster.org). This mutation had been previously described in homozygosis (17) and in compound heterozygosis (4) in two AS subjects confirming its pathogenic role. The mutation lies on exon 6 of the gene transcript AAAS-1 (NM_015665.6), which is absent in the AAAS-2 isoform (NM_001173466.1).

RT-PCR data from patient's fibroblasts showed a significant downregulation of AAAS-1 and an upregulation of AAAS-2 transcripts. Overexpressing AAAS-1 in patient's fibroblasts did not revert the AAAS-2 upregulation. Transcript analysis was performed on RNA extracted from frontal cortex, cerebellum, and spinal cord. The AAAS-1 transcript was significantly downregulated in patient's tissues, while no difference was observed for AAAS-2 expression levels. Treating patient's fibroblasts with cycloheximide did not restore isoform 1 expression or aberrantly-spliced transcript. (Figure 2, Suppl. Figure 1)

\section{$\underline{\text { Protein studies }}$}

The two transcript isoforms are predicted to produce a $\sim 56 \mathrm{kDa}$ protein. To validate the ability of the antibody to recognize the correct band at WB, fibroblasts were transfected with an siRNA targeting Aladin transcripts (ThermoFisher) and harvested after 48 hours. The silencing efficiency was confirmed by a reduction of more than $50 \%$ in both transcripts (Figure 2, Suppl. Figure 1) and by a significant downregulation of the expected band in WB analysis, validating the specificity of the antibody.

While Aladin protein was slightly reduced in total lysates from patient's fibroblasts, a significant reduction was present in patient's frontal motor cortex, cerebellum, and spinal cord. To investigate whether the mutation would interfere with the expected Aladin localization, fibroblasts and tissues were fractionated. Cytoplasmic, nuclear and membrane fractions were analyzed by WB. Aladin was 
detected mainly in the membrane fraction, with a significant reduction in patient's central nervous system tissues and, to a lesser extent, in fibroblasts.

Moreover, the protein distribution among fractions showed an increased cytoplasmic localization in patient's fibroblasts.

\section{$\underline{\text { Cellular studies }}$}

Immunofluorescence analysis showed a perinuclear staining of Aladin in control as well as in patient's fibroblasts, although in AS cells the staining appeared more diffuse in the cytoplasm (Figure 3).

\section{$\underline{\text { Neuropathological studies }}$}

Severe atrophy of the cerebellum, both of hemispheres and vermis, and of prefrontal and motor cortices were observed at a macroscopic level during post-mortem examination.

In the cerebellar cortex, the amount of Purkinje cells was severely reduced, while neurons of the molecular and granular layers were spared. Analysis of primary motor cortex showed a marked loss of pyramidal motor neurons in layers $\mathrm{V}$ and VI, whereas other neuronal subpopulations were unaffected. (Figure 4, Suppl. Figure 2)

In controls, immunostaining with Aladin antibody showed an intense nuclear membrane signal in motor and non-motor neurons in primary frontal cortex. Conversely, patient's neurons showed a marked reduction in staining of the nuclear membrane and occasional spotted cytoplasmic signal. In control cerebella, Aladin signal was localized to the nuclear membrane of Purkinje cells and of neurons in other layers, while in AS samples Aladin was lost in Purkinje cells and markedly reduced in neurons in molecular and granular layers. 


\section{Discussion}

While AS is a heterogenous disorder with respect to both age of onset and phenotypic spectrum, several late-onset cases seem to show a predominant neurological involvement (6). The case described here displays a very late disease onset and a main neurological presentation. The mutation identified in this case lies on the exon spliced out in one of two transcript isoforms of the AAAS gene. As this mutation maps to exon 6, which is present in the AAAS-v1 transcript, it appears to affect protein levels deriving from this isoform, while leaving tissues which predominantly express isoform 2 less affected. Interestingly, other AS cases with mutations within the same exon show an unusual late-onset presentation, as well as the two previously reported cases (onset at 28ys and 25ys) presenting the p.R155H (17)(4). This suggests that a later onset and predominantly neurologic phenotype may be ascribed to a specific genotype. In this respect, reporting clinical information of AS cases and related mutations may aid in the definition of a genotype-phenotype correlation.

To further characterize the AAAS mutation identified herein, we analyzed the expression of AAAS transcripts and the levels of Aladin protein. The c.464G>A mutation is predicted to lead to a missense p.R155H change at the protein level. However, patient’s fibroblasts and tissues displayed a reduction of AAAS-v1 transcript expression. This finding opens two possible scenarios: either the c.464G $>$ A substitution induces an aberrant transcription with intron retention, that eventually undergoes degradation through NMD, or it may increase the affinity of the mutated transcript for RNA interference molecules (18). The lack of transcript changes, after NMD-inhibition with cycloheximide, makes the first hypothesis less probable. Further experiments will explore the role of small interference RNAs as possible causes of transcript 1 downregulation. On the other hand, patient's fibroblasts displayed marked AAAS-v2 over-expression. This result may suggest the importance of isoform 2 in fibroblasts (AAAS-v2 basal levels are higher also in control fibroblasts 
with respect to other tissues) or could be explained by a compensatory mechanism operating in dividing cell lines in vitro.

At the protein level, the mutation is expected to replace a highly conserved arginine with a histidine. The region surrounding the 155-arginine residue belongs to a WD-repeat domain, known to form a typical $\beta$-propeller structure involved in protein-protein interactions and crucial for many cellular functions. The change is predicted to affect the secondary structure of the WD domain (Suppl. Figure 3). (19). We explored the impact of the c.464G>A mutation on the amount and localization of Aladin in patient's fibroblasts and tissues. After subcellular fractionation, the protein was found mainly in membranes, in line with the expected nuclear membrane localization. In the patient's brain, cerebellum and spinal cord, Aladin was ubiquitously reduced, without significant mislocalization, which can be explained by the reduced transcript expression. Conversely, the normal amount of Aladin in patient's fibroblasts, as shown by WB and IF, can be explained by the marked overexpression of AAAS-v2. The altered Aladin subcellular localization in IF and WB suggests that, in addition to the reduction in protein levels, the missense change may lead to a mislocalization of Aladin. Further studies will be needed to confirm this hypothesis.

This study represents the first neuropathological investigation of a patient with AS. Of note, no neuropathological assessment has been described so far in human and animal models of the disease (20). The specific degeneration of motor neurons and Purkinje cells represents the major finding. Interestingly, the most severe loss of Aladin in the perinuclear region was observed in the same neuronal subtypes, suggesting for the first time a link between the neurodegeneration of pyramidal and Purkinje cells and Aladin mutations. Reasons accounting for the susceptibility of these neuronal subtypes are still unknown. A tentative hypothesis may be that neurons with high transcriptional activity require a highly functional NCT machinery, whose integrity is critical to their survival.

In conclusion, we described the clinical, molecular and neuropathological characterization of a lateonset case of AS with primary neurological phenotype associated to a specific AAAS mutation. We hypothesize a role of this mutation of the nucleoporin Aladin in the degeneration of specific 
neuronal populations. This is of particular note, considering the emerging and fundamental implication of NPC and NCT impairment in several neurodegenerative diseases (14).

In this view, further functional analyses of the mechanisms by which disruption of Aladin and other Nups lead to neuronal loss may shed light on the link between NCT impairment and neurodegeneration.

\section{Materials and Methods}

\section{Clinical evaluation and diagnostic workup:}

The study was approved by local IRB. The diagnostic workup included: brain MRI and CT-scan, needle EMG/ENG, muscle and nerve biopsy. Orthostatic hypotension was assessed by measuring the difference between systolic blood pressure values at baseline after lying flat for 3 minutes and after standing for 2 minutes.

\section{$\underline{\text { Genetic and cellular analyses: }}$}

Informed consent was obtained from the patient. DNA was blood-extracted and used in PCR reactions to amplify genomic regions encompassing all $A A A S$ exons. Sanger sequencing was performed on an ABI 3130 DNA analyzer (Applied biosystems) using the Big Dye Terminator 3.1 chemistry.

RNA was isolated from cells and tissues using the Reliaprep RNA cell miniprep system (Promega) according to manufacturer's instructions. RT-PCR assays were used to evaluate the inclusion of exon 6 within AAAS transcripts. For quantitation, we performed SYBR green qRT-PCR relative quantification analysis on a 7500 Real Time PCR System (Applied Biosystems). The deltadeltaCt method was used to calculate the relative quantification (RQ) values of full-length AAAS transcript 
variant 1 (AAAS-1) and deltaE6 AAAS transcript variant 2 (AAAS-2), after normalization to multiple housekeeping genes (ACTB encoding beta actin and rRNA 18S).

In order to silence Aladin expression, an siRNA (ThermoFisher) was transfected into fibroblasts using Lipofectamine RNAiMAX (Invitrogen) and incubated for 48 hours before collection. AAAS1 was overexpressed with plasmid RC200154 Myc-DDK-tagged (Origene) in patient-derived and control fibroblasts using Lipofectamine 3000 (Invitrogen).

To study the presence of nonsense-mediated mRNA decay (NMD), patient's fibroblasts were cultured with and without treatment with cycloheximide (a known NMD inhibitor) at $100 \mu \mathrm{g} / \mathrm{ml}$ for 6 hours, before mRNA isolation and cDNA synthesis.

\section{Western blot analysis:}

Protein aliquots extracted from fibroblasts and tissues overexpressing wild-type and mutated Aladin were separated on a 4-12\% polyacrylamide gel and blotted on a nitrocellulose membrane (Whatman). Subcellular fractions of fibroblasts and tissues (primary motor cortex, cerebellum, and spinal cord) were obtained using Protein Fractionation Kits (Thermofisher).

Samples were probed with the following antibodies: Anti-AAAS TA808612 (Origene) mouse (1:1000), Actin A2066 (Sigma) mouse (1:1200), Lamp1 AB25630 (Abcam) mouse (1:800), Lamin a/c rabbit 2032S (Cell signaling) (1:800-1000), $\alpha$-tubulin 3873S (Cell signaling) mouse (1:8001:1000) as loading and fractionating controls.

\section{$\underline{\text { Immunocytochemistry }}$}

Immunocytochemistry was performed following standard procedures. Cells were washed with $1 \mathrm{x}$ PBS, fixed with 4\% PFA for 10 minutes at room temperature, and washed in 1x PBS three times. Cells were then incubated in $1 \times$ PBS containing 3\% BSA with AAAS primary antibody (AntiAAAS TA808612 Origene at 1:1000 dilution) overnight at $4^{\circ} \mathrm{C}$. Cells were then incubated in $1 \times$ PBS containing 3\% BSA with secondary antibody (Alexa Fluor 488 ab150105, Life Technologies 
at 1:500 dilution) and DAPI for 2 hours at room temperature. Images were obtained by confocal microscopy using a Leica TCS SP2 (Leica Microsystems).

Neuropathological studies:

Samples from different parts of the brain of the patient and two age-matched controls were formalin-fixed and paraffin-embedded according to standard procedures. For immunohistochemistry, small blocks of cerebral frontal cortex and cerebellum were processed. Analyses were performed on $2.5 \mu$ m-thick tissue sections of representative blocks stained for AAAS (TA808612, Origene) and with hematoxylin and eosin.

\section{$\underline{\text { Statistical analyses }}$}

Experiments involving two biological groups were analyzed by two-tailed unpaired Student's t-test. All experiments were performed in triplicate. Significance $*=p<0.05$.

\section{Acknowledgements}

The authors thank the patient and his family for their essential contributing to the work. We thankfully acknowledge Dr. T. Rizzuti who performed the autopsy of the patient and controls.

\section{Competing interests}

The authors declare that they have no competing interests. 


\section{Authors' contributions}

GB, ADF, DR: design of the study, writing manuscript

GB, ADF, DR, GL, AP, FMC, EF, GMC, AB, SS: molecular analysis

GL, AP, FMC: neuropathological analysis

EF, SB, SC, GPC, NB: clinical evaluation and collection of samples

\section{References}

1. Houlden, H. (2002) Clinical and genetic characterization of families with triple A (Allgrove) syndrome. Brain, 125, 2681-2690.

2. Tullio-Pelet, A., Salomon, R., Hadj-Rabia, S., et al. (2000) Mutant WD-repeat protein in tripleA syndrome. Nat. Gen., 26, 332-335.

3. Allgrove, J., Clayden, G. S., Grant, D. B., et al. (1978) Familial glucocorticoid deficiency with achalasia of the cardia and deficient tear production. Lancet, 1, 1284-1286.

4. Kimber, J. (2003) Allgrove or 4 'A' syndrome: an autosomal recessive syndrome causing multisystem neurological disease. J. Neurol. Neurosurg. Psychiatry, 74, 654-657.

5. Vallet, A.-E., Verschueren, A., Petiot, P., et al. (2012) Neurological features in adult Triple-A (Allgrove) syndrome. J. Neurol., 259, 39-46.

6. Patt, H., Koehler, K., Lodha, S., et al. (2017) Phenotype-genotype spectrum of AAA syndrome from Western India and systematic review of literature. Endocrine Connections, 6, 901-913.

7. Li, X., Ji, C., Gu, J., et al. (2005) Molecular cloning and characterization of AAAS-V2, a novel splice variant of human AAAS. Mol. Biol. Rep., 32, 127-131.

8. Cronshaw, J. M., Krutchinsky, A. N., Zhang, W., et al. (2002) Proteomic analysis of the mammalian nuclear pore complex. J. Cell Biol., 158, 915-927. 
9. Kahms, M., Hüve, J., Wesselmann, R., et al. (2011) Lighting up the nuclear pore complex. Eur. J. Cell Biol., 90, 751-758.

10. Nofrini, V., Di Giacomo, D. and Mecucci, C. (2016) Nucleoporin genes in human diseases. Eur. J. Hum. Genet., 24, 1388-1395.

11. Chou, C.-C., Zhang, Y., Umoh, M. E., et al. (2018) TDP-43 pathology disrupts nuclear pore complexes and nucleocytoplasmic transport in ALS/FTD. Nat. Neurosci., 21, 228-239.

12. Grima, J. C., Daigle, J. G., Arbez, N., et al. (2017) Mutant Huntingtin Disrupts the Nuclear Pore Complex. Neuron, 94, 93-107.e6.

13. Prpar Mihevc, S., Darovic, S., Kovanda, A., et al. (2017) Nuclear trafficking in amyotrophic lateral sclerosis and frontotemporal lobar degeneration. Brain, 140, 13-26.

14. Fox, B. W. and Tibbetts, R. S. (2015) Neurodegeneration: Problems at the nuclear pore. Nature, 525, 36-37.

15. Kim, H. J. and Taylor, J. P. (2017) Lost in Transportation: Nucleocytoplasmic Transport Defects in ALS and Other Neurodegenerative Diseases. Neuron, 96, 285-297.

16. Nousiainen, H. O., Kestilä, M., Pakkasjärvi, N., et al. (2008) Mutations in mRNA export mediator GLE1 result in a fetal motoneuron disease. Nat. Genet., 40, 155-157.

17. Nakamura, K., Yoshida, K., Yoshinaga, T., et al. (2010) Adult or late-onset triple A syndrome. J. Neurol. Sci., 297, 85-88.

18. Valencia-Sanchez, M. A., Liu, J., Hannon, G. J., et al. (2006) Control of translation and mRNA degradation by miRNAs and siRNAs. Genes Dev., 20, 515-524.

19. Smith, T. F., Gaitatzes, C., Saxena, K., et al. (1999) The WD repeat: a common architecture for diverse functions. Trends Biochem. Sci., 24, 181-185.

20. Huebner, A., Mann, P., Rohde, E., et al. (2006) Mice Lacking the Nuclear Pore Complex Protein ALADIN Show Female Infertility but Fail To Develop a Phenotype Resembling Human Triple A Syndrome. Mol. Cell. Biol., 26, 1879-1887. 


\section{Figure legends:}

\section{Figure 1}

Schematic representation of $A A A S$ gene. Vertical bars represent exons. The mutations described so far and the one (red) described in the patient are reported (A). The mutation lies on exon 6, present only in isoform 1 (B) and on the first WD domain at the protein level (C). MTS=microbody targeting signal. Electropherogram showing the homozygous c.464G>A and a WT control (D). Conservation of the arginine in position 155 across different species (E). Patient Pt-R155H pictures showing the furrowed and atrophic tongue (F-1), hypotrophy of interossei muscles and tenar eminence (F-2). Hypotrophy of the posterior (F-3) and anterior (F-4) muscles of distal limbs and pes cavus. Patient T1-weigthed MRI sagittal section showing cerebellar atrophy (F-5). Patient T2weighted MRI axial section showing severe putaminal atrophy (F-6).

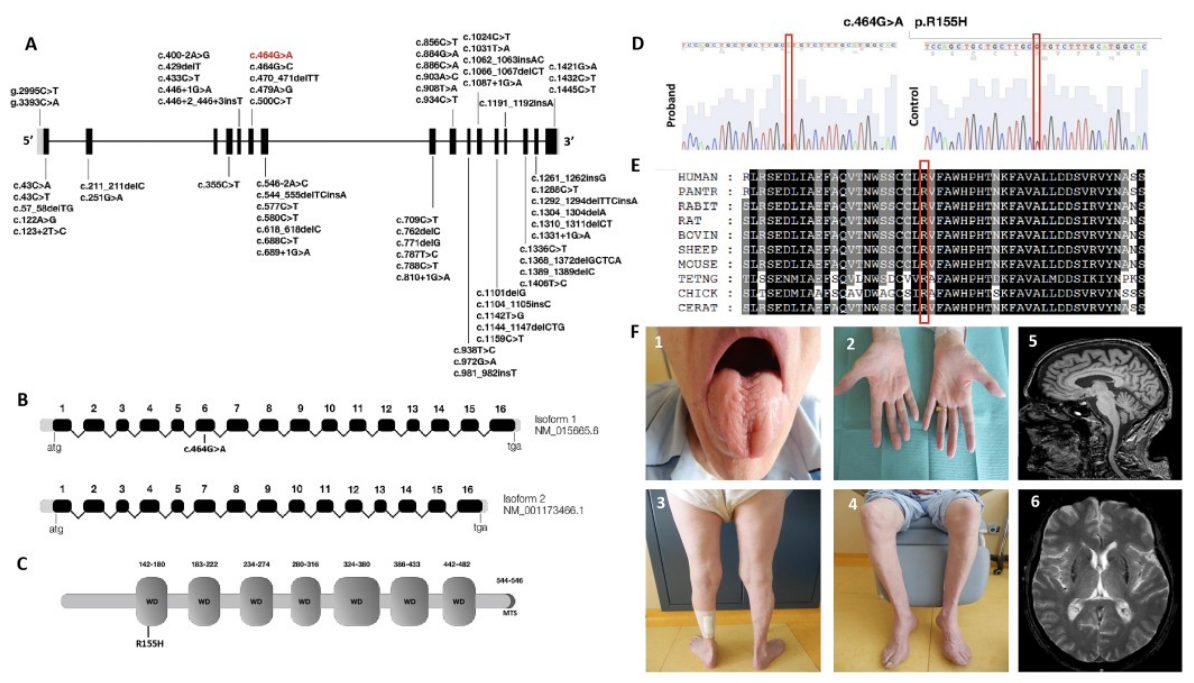




\section{Figure 2}

RT-PCR plot showing the differential expression of AAAS v-1 and AAAS v-2 in fibroblasts (A), primary motor cortex, cerebellum and spinal cord between patient and two controls. WB analyses of Aladin protein in total lysates from fibroblasts (B), primary motor cortex, cerebellum and spinal cord in patient and two controls.
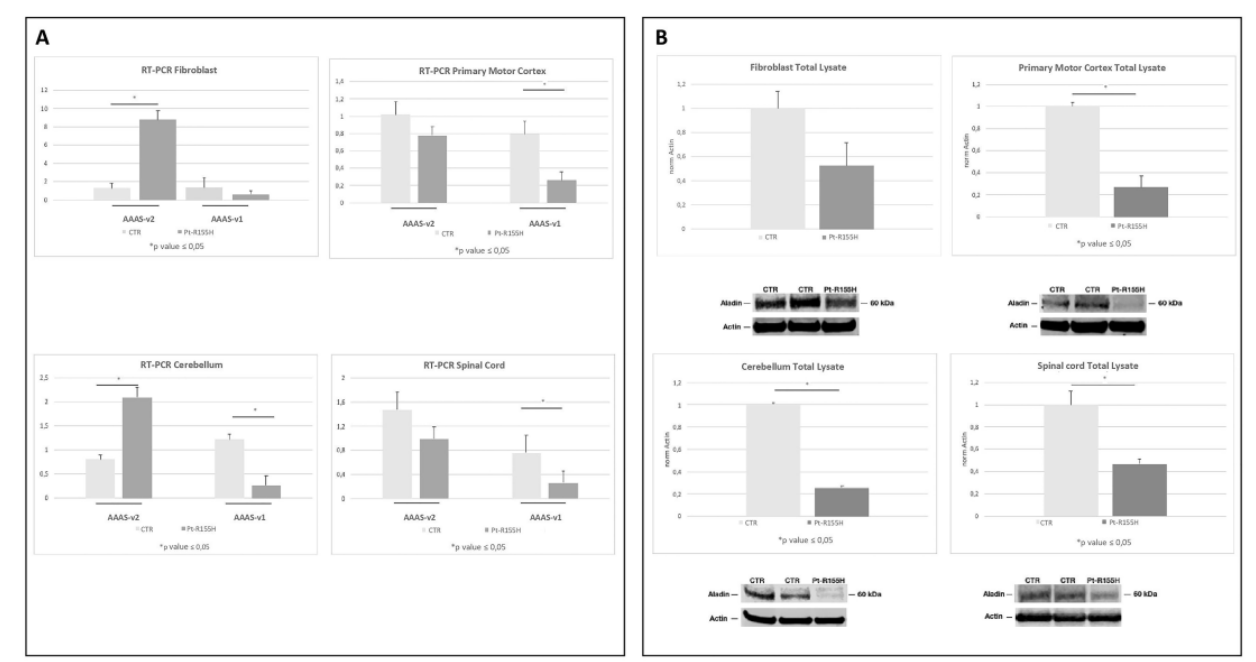


\section{Figure 3}

Immunofluorescence showing Aladin protein (green) and DAPI (blue) in fibroblasts showing the different distribution pattern between patient and controls (A). WB analyses of Aladin protein in subcellular fractions (cytosol, membrane and nucleus, normalized for the respective markers $\alpha$ tubulin, Lamp1, lamin a/c) from fibroblasts (B), primary motor cortex (C), cerebellum (D) and spinal cord (E) in patient and control.
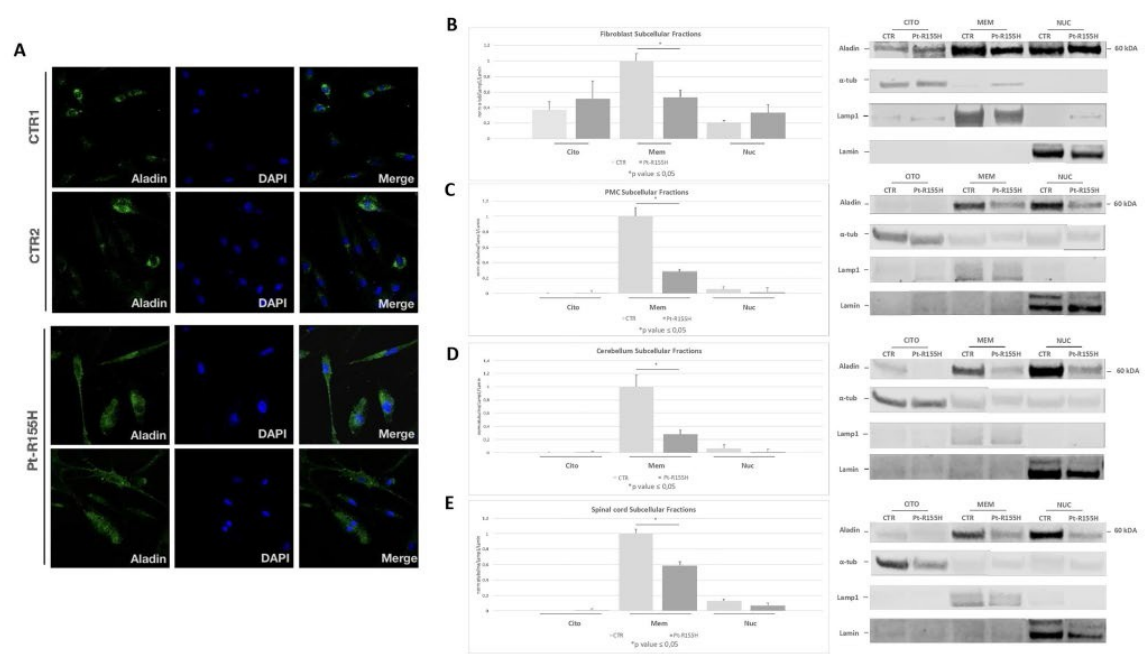


\section{Figure 4}

Hematoxylin-Eosin staining of motor neurons of primary motor cortex and cerebellum from patient and control (A) and the counting of neuronal cells from three independent sections (B), (10x magnification). The image shows a loss of pyramidal motor neurons and Purkinje cells in patient, while the cerebellar cells from granular and molecular layers are not reduced. Immunohistochemistry using Aladin antibody in primary motor cortex showed diminished perinuclear staining in patient neurons and a more diffuse cytoplasmic localization (C), (5x and 20x magnification). A significant reduction of Aladin perinuclear staining is detected in the surviving Purkinje cells as well as in neurons from molecular and granular layers of cerebellar sections (D), (5x and 20x magnification).

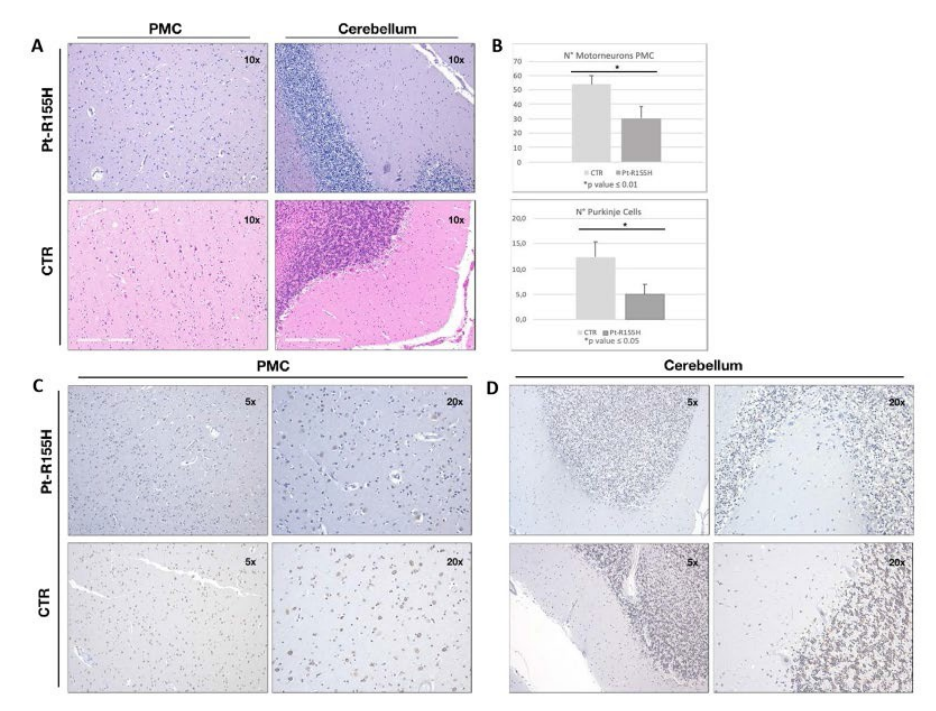




\section{List of Abbreviations}

Allgrove Syndrome (AS)

Nuclear Pore Complex (NPC)

Nucleoporin (Nup)

Nucleo-Cytoplasmic Transport (NCT)

Magnetic Resonance Imaging (MRI)

Electromyography (EMG)

Electroneurography (ENG)

Nonsense-Mediated Decay (NMD) 